\title{
The mysteries of the diagonal: Gender-related perceptual asymmetries
}

\author{
JUDITH AVRAHAMI, TALY ARGAMAN, and DVORA WEISS-CHASUM \\ Hebrew University of Jerusalem, Jerusalem, Israel
}

\begin{abstract}
In this article, we report a perceptual asymmetry for the two diagonals that is related to gender in that females prefer the diagonal spanning from top right to bottom left (/) whereas males prefer the opposite ( $($ ). This relationship is observed in a variety of tasks, including aesthetic judgment of paintings, spotting differences between two paintings, and visual search for a tilted line among similarly tilted distractors. This article does not provide an explanation of the relationship between this asymmetry and gender but rules out several potential mediating factors, such as eye dominance, head tilt, handedness, and hemispheric differences. At the same time, the scope of the phenomenon is outlined: The asymmetry is found for both meaningful and meaningless stimuli and in both brief and extended presentations. Moreover, the asymmetry is found to be related to the tilt of the visual elements that require processing, not to their location in the visual field.
\end{abstract}

Even with little awareness of perceptual phenomena, one might notice a prevalent asymmetry between the two diagonals. Painters such as Jan van Eyck, for example, structure their paintings mostly along one of the diagonals. Thus, in a van Eyck painting furniture, floor boards, and sometimes even people lie along the diagonal spanning from the top right end of the painting to the bottom left end. The painter Jacques Louis David, on the other hand, consistently prefers the opposite diagonal: that from the top left to the bottom right.

Advertisers too tend to favor one diagonal more often than the other. Web sites of shoe vendors are a good example: All shoes are diagonally oriented in one direction in some of them and in the opposite direction in others. Another, quantifiable example can be found in the corpus of line drawings of everyday objects by Snodgrass and Vanderwart (1980). In that corpus, there are a number of elongated objects, such as a screwdriver or a spear of asparagus, all oriented diagonally. Interestingly, those oriented along one diagonal outnumber those oriented along the other by a factor greater than $2: 1$. Even in scientific papers, figures depicting an experimental procedure as a procession of displays a participant would experience over time use one diagonal more often than the other to represent the time sequence. It is important to note, however, that although painters and designers may be found to favor one diagonal over the other, they do not all prefer the same one. It would be interesting, therefore, to find out whether this differential preference is related to any other individual characteristics.

Correspondence concerning this article should be addressed to J. Avrahami, Goldie Rotman Center for Cognitive Science and Education, School of Education, Hebrew University of Jerusalem, Jerusalem 91905, Israel (e-mail: judav@vms.huji.ac.il).
The asymmetry between the two diagonals is particularly intriguing since it cannot be reduced to asymmetries between the right and left hemifields (see, e.g., Kosslyn, 1987) or between the upper and lower hemifields (see, e.g., Christman, 1993; Rubin, Nakayama, \& Shapley, 1996), both of which have been studied extensively. The two diagonals span both the right and left visual fields and both the upper and lower fields.

Still, like any perceptual asymmetry, if the asymmetry between the diagonals is experimentally established it would have important implications for the understanding of various aspects of visual processing, such as efficiency and preference. In particular, the asymmetry would illuminate the temporal component of the visual process: Assuming that the more efficiently processed parts of a stimulus reach a threshold of activation faster than others, it would indicate which aspects of a stimulus have precedence in visual processing - that is, which aspects have a more important role in constraining the interpretation of a visual scene.

There are at least three possible ways to conceive of precedence in visual processing. One involves a scan along the display with attention (and/or the eyes) moving from one end of the display to the other, the second relates to a differential perceptual accessibility of the content in different locations in the visual field, and the third corresponds to greater featural availability or higher salience of one type of stimulus over another. The set of experiments reported here is an attempt to characterize the asymmetry and relate it to one of these classes.

The study comprises three experiments in which the perceptual asymmetry between the two diagonals was tested. In the first experiment, we compared aesthetic preference for paintings having one diagonal structure or the other and for mirror images of the same paintings. In the second experiment, we tested whether or not flipping 
a painting to its mirror image affects the order in which differences are detected in a "spot-the-differences" task. In the third experiment, we compared the efficiency of a visual search for lines tilted to the right or to the left of vertical under either extended or brief presentation. To anticipate, all of the experiments reveal the asymmetry to be related to gender.

\section{EXPERIMENT 1}

Since much of the evidence for the asymmetry of the diagonals comes from art, we first wanted to find out whether people have a preference for paintings whose dominant diagonal is the one or the other. To this end, we asked participants to aesthetically compare paintings with a dominant diagonal with their mirror images. Thirty-two paintings, half originally structured along one diagonal and half along the other, were used. Each painting was printed on one side of a sheet of paper, and its mirror image was printed on the other side. The participants were asked to indicate which version they liked better. The experimental question was whether the participants would tend to prefer the original irrespective of the orientation of its dominant diagonal, or whether preferences would be mediated by the layout. An example of the paintings used in the experiment is presented in Figure 1.

\section{Method}

Participants. Forty-eight students of the Hebrew University, half of them male and half female, participated in the experiment for a small monetary reward. A quarter of both the male and the female participants were left-hand dominant.

Stimuli. The corpus of paintings was comprised of four groups of eight: paintings focusing on a single male, paintings focusing on a single female, paintings of landscapes, and paintings of assemblies of people. The orientation of the dominant diagonal was balanced within each group of paintings. A pretest ensured that observers agree with our perception of the dominant diagonal in each painting. The paintings were fitted to the size of an A4 page, with the original printed on one side and its mirror image on the other. The paper was then laminated to avoid wear and tear. The paintings were all realistic, dating from 1510 to 1928 .

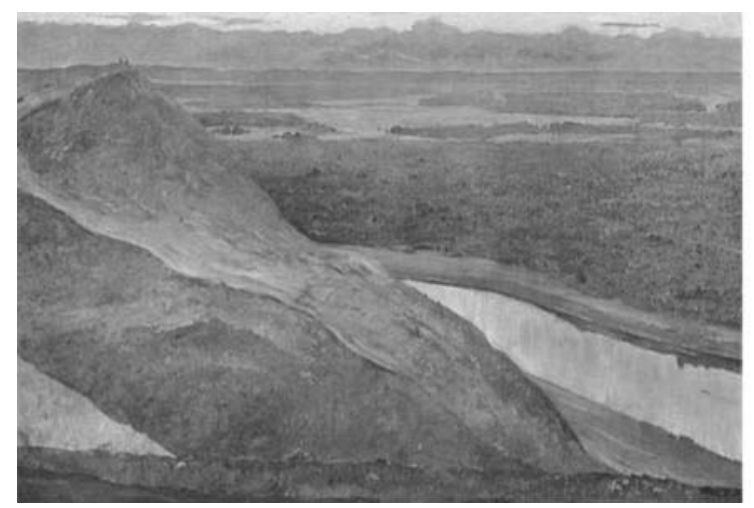

Procedure. The participants were presented with one painting at a time, in random order. They flipped the sheet back and forth until they made up their minds as to which side they liked better. The experimenter recorded the following information: (1) which version of a painting (original or mirror image) the participants happened to see first and (2) which version of a painting the participants preferred. For control, the experimenter also recorded each participant's gender and handedness. The experimenter was unaware of which version was the original. The participants enjoyed the paintings and the task.

\section{Results}

Every choice of each painting was given a score of +1 if the original version was chosen or -1 if the mirror image was chosen. This revealed that the original version of the painting was preferred in $52.9 \%$ of the cases. The scores were then subjected to an analysis of variance (ANOVA) with painting as the unit of analysis and with dominant diagonal (of the original) and participants' gender as factors. The analysis revealed that the interceptthat is, the preference for the original over its mirror image-deviated significantly from zero $[F(1,46)=$ $\left.7.526, M S_{\mathrm{e}}=0.044, p=.009\right]$. This preference may indicate that some of the participants had seen a few of the paintings before-even if unaware of it - or it may possibly indicate that the original does, somehow, look better. ${ }^{1}$ There was also a slight tendency to prefer the original more often when it was structured along the topright-to-bottom-left [henceforth TR-to-BL (/)] than along the top-left-to-bottom-right [henceforth TL-to-BR $(\backslash)$ diagonal, but the ANOVA shows that the effect of dominant diagonal is not significant $[F(1,46)=2.157$, $\left.M S_{\mathrm{e}}=.074, p=.149\right]$.

The analysis did reveal a significant interaction between dominant diagonal and gender $[F(1,46)=5.465$, $\left.M S_{\mathrm{e}}=.074, p=.024\right]$. Females were found to prefer the original more often when it was structured along the TRto-BL (/) diagonal, whereas males preferred the original more often when it was structured along the opposite diagonal ( $\backslash$; see Figure 2). The percentages of cases in which the original was chosen by males for each diagonal are $53.6 \%$ and $56.0 \%$ for $(/)$ and $(\backslash)$, respectively, and

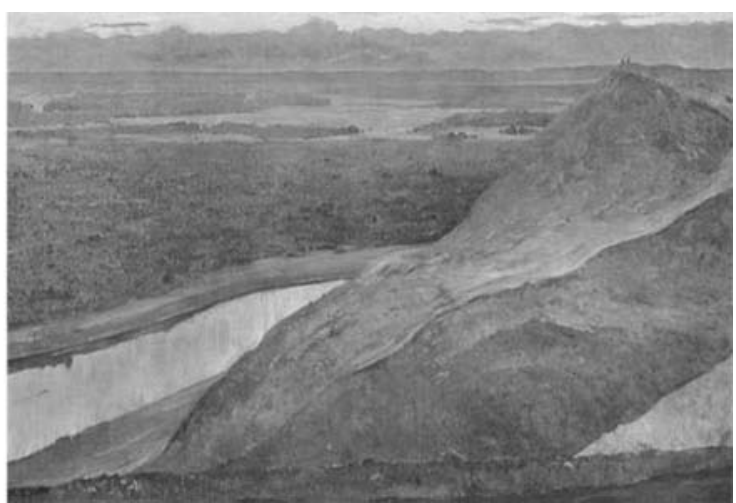

Figure 1. Example of a painting and its mirror image used in Experiment 1 (Mikhail Nesterov, Homeland of Aksakov, 1914). 


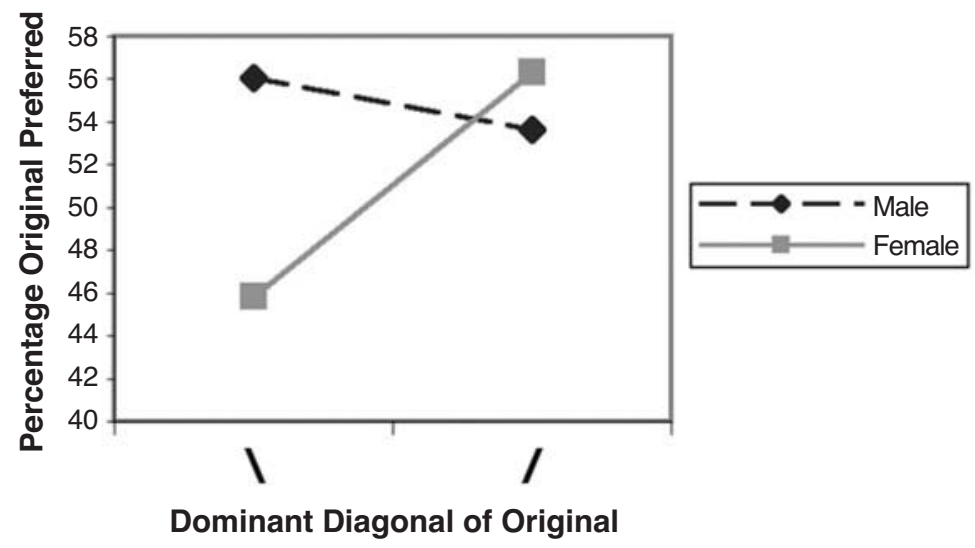

Figure 2. Percent of cases in which the original, in each diagonal, was preferred over its mirror image by the males and the females.

the percentages for females choices are $56.3 \%$ and $45.8 \%$ for $(/)$ and $(\backslash)$, respectively.

Yet another analysis, this time including handedness as a factor as well, did not reveal any new effects. Neither the interaction between handedness and diagonal nor that between handedness, gender, and diagonal was found to be significant (both $F \mathrm{~s}<1$ ).

The interaction between dominant diagonal and gender came as a surprise. We therefore tried to explain it away by a difference in attitude toward the orientation of the body of the same or the opposite sex, specifically in the single-male and single-female groups of paintings. An additional analysis, with the four groups of paintings as another factor, showed a similar pattern in all four groups of paintings (for the triple interaction of diagonal, gender, and groups, $F<1$ ).

Another explanation of the interaction that had to be checked is that, possibly, what the participants actually preferred was the version they had seen first. Since the version of the painting seen first was randomly determined, it could be that, by chance, the males happened to see first more original versions oriented along the TLto-BR $(\backslash)$ diagonal whereas the females first saw more original versions oriented along the other diagonal; such a difference could explain the gender-related difference in preference. Indeed, a slight correlation (.147 for males and .167 for females) was evident between the version seen first and the preferred version. The percentages of participants of each gender who happened to first see the original version in pairs of paintings of each dominant diagonal orientation are as follows: With the TL-to-BR ( ) dominant diagonal, both the males and females more often saw an original first ( $55 \%$ for the males, $51 \%$ for the females). With the TR-to-BL (/) dominant diagonal, the males saw the originals first as often as they saw mirror images first (i.e., 50\%), and the females saw originals first slightly less often (49\%). These values cannot explain the pattern of results: Although order of presentation could contribute to the pattern of male preferences, it can explain neither the difference in preference evident in the females nor why the difference in the females is the larger of the two.

\section{Discussion}

Although the result of differential preference in the different genders was, as we said, a surprise, it solved a puzzle that had preoccupied us before. When searching the literature for treatments of the diagonals in the theory of art, we found two art historians who considered the diagonal in paintings. Both argued that the experience derived from looking at a painting changes in an important manner when, instead of viewing the original, one views a mirror reflection of it.

One of these art historians, Rudolf Arnheim, argued in his book, The Power of the Center: A Theory of Composition in the Visual Arts (1982):

There is a well known tendency, largely independent of actual eye movements, for viewers to perceive the area in the left corner of the visual field as the point of departure and the entire picture as organized from left to right. Also contributed by the viewer is a downward pull ... (p. 37)

This obviously results in a TL-to-BR $(\backslash)$ organization. To prove his argument, Arnheim presented J. L. David's painting Napoleon Crossing the Alps, in which Napoleon's horse appears to halt, and commented, "If one looks at David's painting in the mirror one sees the horse bounding much more freely" (p. 108). With the question of an active scan set aside for the time being, it is obvious that Arnheim considered the TL-to-BR $(\backslash)$ diagonal as primary in viewing a painting.

Another art historian, who considered what she called the "glance-curve," is Mercedes Gaffron (1950). She argued that the glance-curve "begins in the left foreground, penetrates toward the depth, then turns over toward the right" (p. 317). Although Gaffron spoke of the three-dimensionality of a painting, she clearly sketched a path consistent with the TR-to-BL (/) diagonal that later 
curves down. To prove her point, she presented several paintings and their mirror images. One of the paintings is Pope Leo $X$ by Raphael. In the original, according to Gaffron, objects lying along the path from the bottom left toward the top right (/), such as the pope's illuminated manuscript and elaborate bell, are seen as related to the pope's face. In the mirror image, she argued, they can only be seen separately, as a still life, "but we cannot perceive them in immediate connection with the face of the Pope, and thus they lose the special meaning they have within the composition of the original" (p. 323).

Arnheim (1982) and Gaffron (1950) both believed in a diagonal path of the viewer's glance, but they differed in which diagonal it is. Interestingly, Gaffron, a woman, argued for the diagonal preferred by the females in our experiment, whereas Arnheim, a man, advocated the diagonal preferred by the males!

To further understand the phenomenon on our hands, we asked whether the gender-related differential preference for diagonals was restricted to aesthetic judgment or was more generally related to perceptual processing. If flipping a painting to its mirror image changes the way the painting is perceived, then parts that are salient in one layout may lose their salience in the other, and vice versa. ${ }^{2}$

To test this hypothesis, we used the well-known "spotthe-difference" game to compare performance with paintings and with their mirror images. We reasoned that if a change in layout changes the processing of a painting, then differences that are spotted before others in the original might be spotted later in its mirror image.

\section{EXPERIMENT 2}

Ten paintings were selected for the experiment, and two changes were made in each. Observers watched the intact and changed versions of a painting displayed side by side on a computer screen. When they spotted a difference, the observers indicated its location by pressing the computer's mouse key.

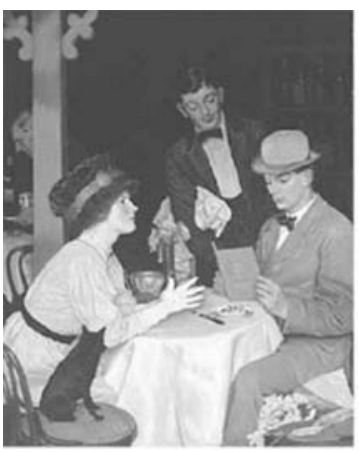

A

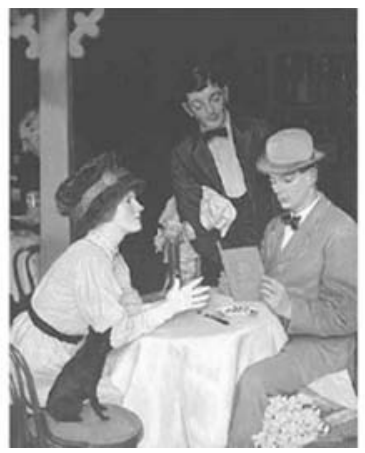

B

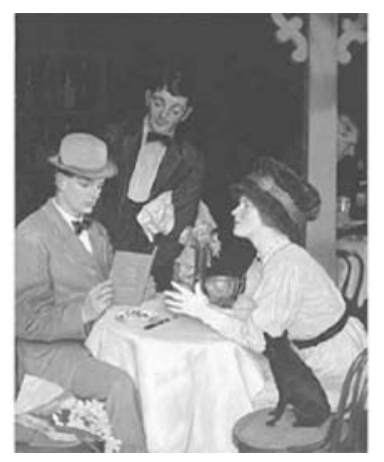

C

\section{Method}

Participants. Fifty-two students, half of them male and half female, participated in the experiment for a monetary reward.

Materials and Procedure. Ten classical paintings with asymmetric structures were used. In each painting, one of the diagonals was more salient than the other. Two changes were introduced into each painting. The differences between the original and the modified versions were neither very easy nor too difficult to spot. This level of difficulty was reached after extensive pretesting. The locations of the changes in the paintings were varied, to avoid learning. For every pair of intact and modified paintings, there were two versions: the original orientation and a mirror image of it. For an example, see Figure 3.

Every participant spotted the differences of a painting either in its original pairs or in the mirror image pairs. In addition, half of the pairs presented to each participant were structured along one diagonal and the other half along the other diagonal.

The paintings were presented on the monitor of a portable Toshiba Satellite computer. A computer program presented the paintings in random order. The assignment of the intact painting and the changed version to the left or right half of the computer monitor was also random. The program recorded the time when the mouse key was pressed in the vicinity of a difference. The participants could point the mouse and click its key at either the changed or the intact version of the painting in a pair, as long as it was in the area of a difference between the versions.

Design. The two variables manipulated in the experiment were layout - that is, the basic structure of the versions of the painting in a pair (which changes, of course, in the mirror image) - and gender. For every painting, each layout was presented to half of the male and half of the female participants. The dependent measure was the order in which the differences were spotted - that is, which of the two differences was spotted first.

\section{Results}

At this stage, we still did not know what the effects of flipping a stimulus to its mirror image would be. Moreover, since, as was indicated above, the locations of changes made to the paintings were varied in the different paintings, we had no theory as to which change would be spotted first and by which group. All we tested here is the hypothesis that whatever effect the flipping from one layout to the other would have on the females' processing, it would have the opposite effect on that of the males.

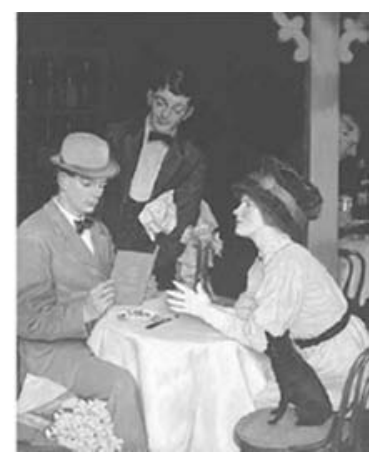

D

Figure 3. Versions of William Strang's painting Bank Holiday (1912) used in the task of spotting two differences made in the original and in a mirror image. (A) original version; (B) original version with two differences; (C) mirror image; (D) mirror image with two differences. 
Looking at the spotting order of the female group for the two layouts, we found a difference. This difference could, naturally, be a matter of chance. To establish an interaction between layout and gender, we must see the opposite difference in the male group. In other words, changes that are more likely to be spotted first in one layout over the other by females should also be more likely to be spotted first in one layout over the other by males - but for them this should occur in the opposite layouts.

To find out, we scored the order of finding the two differences as " 1 " if it matched the order in which the females tended to spot these differences when the paintings were in the version with the TR-to-BL (/) layout and " -1 " if it did not. Thus, every trial (i.e., every completion of spotting the two differences in a painting) received a value of either 1 or -1 depending on the order in which the differences were spotted. We then calculated the mean value of every painting separately for either layout and for either gender (see Figure 4).

The mean values for spotting the differences are .046 and -.076 for the females for the $(/)$ and the $(\backslash)$ diagonals, respectively, and -.046 and .200 for the males for the $(/)$ and the $(\backslash)$ diagonals, respectively.

Note that whereas for the females the value is higher in the TR-to-BL (/) diagonal than in the other, the opposite is true for the males - namely, the value is higher in the TL-to-BR $(\backslash)$ diagonal than in the other. An ANOVA of spotting order for the 10 paintings with layout and gender as factors revealed a significant interaction between the two factors $\left[F(1,9)=13.505, M S_{\mathrm{e}}=.006, p=.005\right]$.

One could argue, of course, that the interaction could have resulted from the way we scored order. Therefore, two planned comparisons, one for the females and one for the males, were conducted. The effect of layout in the separate analysis for females is $F(1,9)=10.288, M S_{\mathrm{e}}=.002$, $p=.011$, but this, again, could be related to the way we scored order. Importantly, however, the separate analysis for the males again revealed a significant effect of layout on spotting order $\left[F(1,9)=6.000, M S_{\mathrm{e}}=.013, p=.037\right]$, with the effect being opposite to that on the females' spotting order (see Figure 4). Unlike the previous significant results - namely, the interaction between layout and gender and the effect of layout for the females only - this result cannot be attributed to the method of defining the spotting order.

\section{Discussion}

The results of this experiment clearly demonstrate an effect of layout on the temporal aspect of perception. Even though some differences were, naturally, more meaningful, more salient, and hence easier to spot than others, this saliency was still affected by the structure of the paintings: A difference that was more salient than another in one layout could be less so in the mirror image of the same painting. Most important, the effect of layout on the salience of a difference worked in opposite directions for the males and the females.

We have, by now, two pieces of evidence indicating a gender-related perceptual difference, but it is not clear why flipping of a painting to its mirror image affected either the aesthetic pleasure derived from the painting or the order in which the differences were spotted. There are two possible explanations for this effect. One is related to locations in the visual field: If certain locations are more accessible than others - and differently so to males and to females - then, since parts of a painting switch locations when the painting is flipped, any part of a painting can become more accessible or less so in the mirror image. As a result, elements that are meaningful in one layout may become, as Gaffron (1950) argued, meaningless in a mirror layout, and the painting less coherent, less harmonious. The same elements may also become less salient and, hence, changes introduced in them more difficult to spot. Differences in the accessibility of

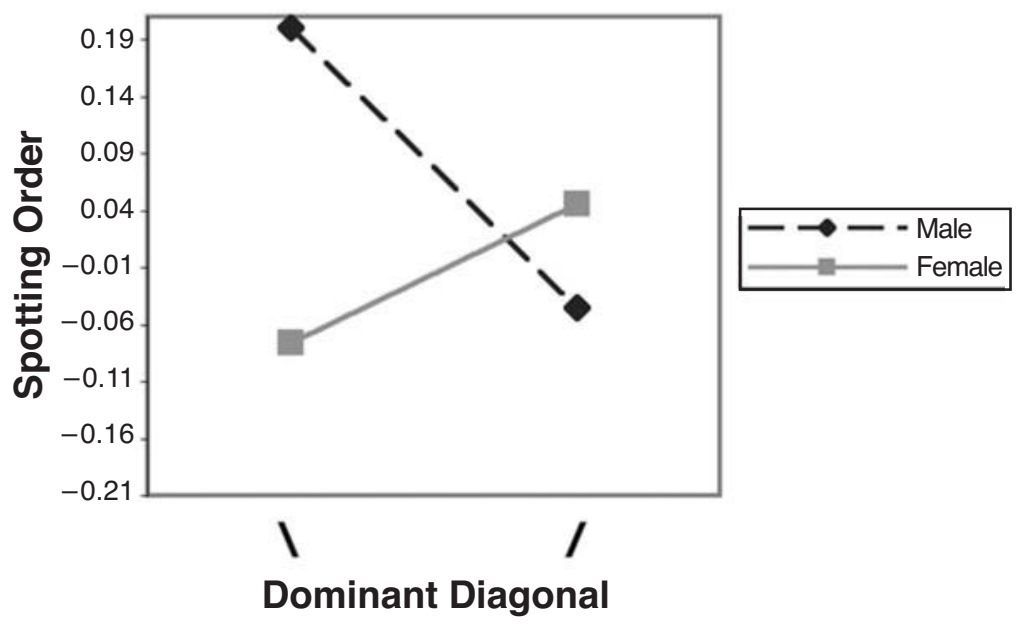

Figure 4: Mean value for spotting order. Spotting in the order in which the majority of females spotted in the TR-to-BL (/) layout is scored " 1 ," whereas spotting in the opposite order is scored " -1 ." 
locations in the field could (but would not necessarily) result from a scan of the display (this issue is addressed in more detail in the General Discussion section). Another possible explanation is that there is a differential sensitivity to orientation in males and females. Since flipping a painting to its mirror image changes the orientation of its elements, elements that are more salient in one orientation may become less so in the other. This, in turn, may again affect both the coherence of a painting (and hence its aesthetic experience) and participants' efficiency in spotting differences. Note that the two explanations are nonexclusive. Experiment 3 was designed to test the two hypotheses in a fully controlled display.

\section{EXPERIMENT 3}

The main goal of Experiment 3 was to test the location hypothesis versus the orientation hypothesis. To that end, the display in this experiment consisted of 36 short lines, all tilted either to the right or to the left of vertical. In half of the trials, 1 of the lines, serving as a target, was tilted less than the rest. The participants searched for a target and judged whether or not one was present. The possible locations of the target were confined to a circle around the center of the display and were fully balanced.

To test the location hypothesis, we could ask whether or not efficiency in detecting a target in locations along the two diagonals (relative to display center) is differentially related to gender. To test the orientation hypothesisthat is, that males and females differ in their sensitivity to the orientation of elements in the display-we could ask whether males and females differ in the ease with which they detect targets oriented in different directions, irrespective of their location.

Another function of Experiment 3 was to find out whether the asymmetry related to gender is in fact mediated by other individual characteristics. The individual characteristics recorded for every participant, in addition to gender, were eye dominance, head tilt, and handedness. The experiment also enabled us to explore the relation between gender and perceptual sensitivity in the left versus the right hemifield. We could thus also test whether hemispheric differences can account for the relation, found in the previous experiments, between gender and diagonal.

In addition, in the experiment we tested a number of questions concerning the scope of the asymmetry.

A preliminary question was whether the perceptual asymmetry, found with rich and meaningful stimuli in the two previous experiments, would be at all evident with poor and meaningless stimuli. The stimulus in this experiment consisted of just a group of short tilted lines.

Two additional questions were whether the asymmetry is slow to build, requiring a long presentation that allows free eye movements, and whether the asymmetry can be overcome by adjusting to a repeatedly presented stimulus tilted always in the same direction.

Concerning the individual characteristics that were recorded here, three conditions must hold to truly ex- plain the gender-related perceptual asymmetry: (1) the relation between gender and diagonal must be replicated in the new experiment, (2) the individual characteristics must be more strongly related to the asymmetry than gender is, and (3) these characteristics should be correlated with gender. If, however, only the second condition holds, it could at least provide a potential explanation for the gender asymmetry found in the first experiments, under the (not very likely) assumption that the gender groups in those experiments were unbalanced in one of the other individual characteristics.

Experiment 3 consisted of two subexperiments. In one, presentation lasted until the participant responded, whereas in the other presentation was brief.

\section{Experiment 3A}

In Experiment 3, participants searched for a target among distractors in a display consisting of 36 short lines. Both target and distractors were tilted in the same direction, but the distractors were tilted more than the target.

\section{Method}

Participants. Sixty-two students of the Hebrew University participated in the experiment, some for a monetary reward and others in partial fulfillment of course requirements. Two participants were excluded from the analyses: 1 female whose performance was at chance and the weakest male (to match the number of participants in the two gender groups). Half of the participants were female, and the other half were male. Tests revealed that of the 60 participants 38 were right-eye dominant, 49 were right-hand dominant, 23 had a rightward head tilt, 13 had a leftward head tilt, and 24 did not show any tilt.

Materials. Thirty-six lines, all tilted either to the right or to the left of vertical, were presented within an imaginary grid of $6 \times 6$ centered on a computer screen. Each line subtended $.6^{\circ}$ of visual angle, was 2 points thick (produced with anti-aliasing), and was randomly positioned with a cell of the imaginary grid subtending $1.3^{\circ} \times 1.3^{\circ}$ of visual angle. The whole imaginary grid subtended $8^{\circ} \times 8^{\circ}$ of visual angle. All distractors were tilted $45^{\circ}$ away from vertical, whereas the targets were tilted only $30^{\circ}$ (see Figure 5). There were eight possible locations for targets, as indicated in Figure $5 \mathrm{C}$, all equally distant from the center of the display.

The visual search task was run on a Mac IICX computer with a 13-in. monitor and was controlled by the VScope software package (Enns \& Rensink, 1992).

In addition, a paper cone was used to determine eye dominance, and a note stand balanced by a level was used to determine head tilt.

Procedure. On entering the laboratory, the participant took a seat facing the experimenter and was asked to read the instructions that were placed on the note stand. Meanwhile, the experimenter used the vertical center bar of the note stand, extended above the instruction sheet, to determine the participant's head tilt.

The participant then turned to face the computer and perform the visual search task. This task consisted of five blocks in all. The first was a short familiarization block of 8 trials, in which orientation of the display was mixed (i.e., the lines were tilted to one side in some trials and to the other side in other trials). The second block, which was the practice block, consisted of 64 trials and was again mixed. The three experimental blocks, each consisting of 64 trials, then followed. The first two experimental blocks were pure blocks - that is, in all trials within a block lines were tilted to one side. Each of the pure blocks had either a right tilt or a left tilt, and their order was balanced across the participants of each gender. The third experimental block was mixed, like the practice block. 


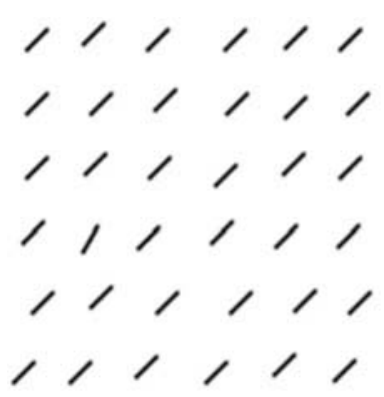

A

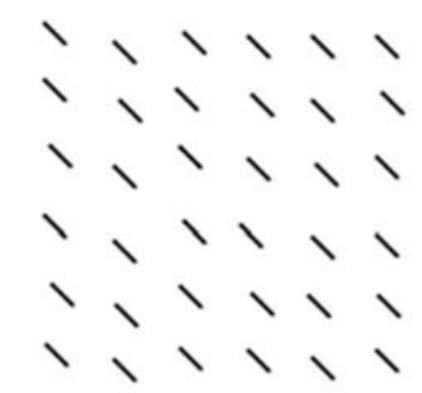

B

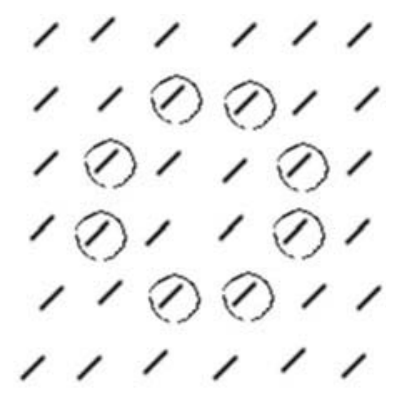

C

Figure 5. Examples of the display used in Experiment 3: (A) target present, tilted right; (B) target absent, tilted left; (c) potential target locations.

The schedule of a single trial was as follows: The experimental display was presented until a response was made. If no response was made within $3.6 \mathrm{sec}$, time-out was reached. After a blank screen of $75 \mathrm{msec}$, the feedback- "+" for correct, "_" for incorrect, and " 0 " for time-out - appeared at screen center for $675 \mathrm{msec}$. A 750-msec blank screen preceded the next experimental display.

The participant pressed a key with the left hand in target-present trials and with the right hand in target-absent trials.

When finishing the visual search task, the participant was asked to look at the experimenter through the paper cone, focusing on the experimenter's nose. This enabled the experimenter to determine the participant's dominant eye.

Design. Three within-participants variables were manipulated: the orientation of the lines of the stimulus (left vs. right of vertical), target presence (present vs. absent), and the location of the target. Obviously, the third variable was manipulated only within targetpresent trials. Analyses therefore always involved only two of these variables: orientation and presence collapsing over target location, and orientation and location for target-present trials only. Locations were collapsed in two ways: top-right and bottom-left locations versus top-left and bottom-right locations, and the four pairs of locations at the ends of both diagonals.

Other variables whose effects on performance were tested were gender, eye dominance, head tilt, and handedness.

\section{Results}

Separate analyses were carried out for the pure and the mixed experimental blocks. The pure blocks were more useful for testing the locations hypothesis, since the factor of location was not confounded with the orientation of the stimulus, which was fixed. In contrast, the mixed block was most useful for testing the hypothesis of object orientation.

Pure blocks. The two pure blocks were lumped together, and an ANOVA with line orientation, target presence, and gender as factors was conducted both for accuracy and for speed. In this and the following experiment, analyses for speed involved median response times (RTs) for correct responses only. The only significant effects were revealed by the analysis of RT. One was a main effect of target presence, with response being faster $\left[F(1,58)=53.42, M S_{\mathrm{e}}=43,083, p<.001\right]$ when a target was present. This finding is common in visual search tasks. The other was a main effect of gender: The males were faster than the females $[F(1,58)=5.59$,
$\left.M S_{\mathrm{e}}=198,648, p=.021\right]$. There was no main effect of orientation and no interaction between stimulus orientation and gender (both $F_{\mathrm{S}}<1$ ). The analysis for accuracy revealed no significant effects at all. At this stage, it was unclear whether the interaction between orientation and gender was not found because the stimuli were meaningless, because the pure blocks enabled adjustment to stimulus orientation, or because the previously found gender-related diagonal asymmetry is related to location and not to element orientation.

Further analyses of the data of the pure blocks, for both speed and accuracy, focused on target location in the target-present trials only, for a comparison of efficiency in the different diagonals of locations.

In the analyses, efficiency in the four locations at the ends of one diagonal (e.g., the two upper right and the two lower left locations in Figure 5C, representing the TR-toBL diagonal of locations) was compared to that at the ends of the second diagonal, to answer the following questions:

1. Would processing of locations along one diagonal be more efficient than processing along the other?

2. Would gender and locations - on the different diagonals-interact?

3. Would targets of one orientation be more efficiently detected in locations along one diagonal than in locations along the other diagonal?

ANOVAs were conducted with orientation and diagonal of locations as within-participants variables and gender as a between-participants variable, both for accuracy and for speed. None of the new potential effects reached significance. The main effect of gender for speed reappeared here, with the males being faster than the females, and a main effect of diagonal of locations for accuracy almost reached significance $\left[F(1,58)=3.929, M S_{\mathrm{e}}=\right.$ $21.914, p=.052]$, showing a small $(1 \%)$ but consistent advantage of the TR-to-BL (/) over the TL-to-BR ( $($ ) diagonal of locations. Most important, performance in the two diagonals of locations did not interact with gender $(F<1)$. There was only a hint of an interaction between orientation and diagonal of locations for speed $[F(1,58)=$ $\left.1.94, M S_{\mathrm{e}}=5,331, p=.169\right]$, with the participants being 
slightly faster when orientation matched the diagonal of locations than when it did not.

Since no effect of either orientation or diagonal of locations (or their interaction) was found to be related to gender, there was nothing to be explained, at this stage, by other individual characteristics; therefore, no further analyses were conducted on the data of the pure blocks.

Mixed block. Analyses of the mixed experimental block were then conducted. First, analyses for accuracy and for speed were conducted with orientation, presence, and gender as factors. These analyses again revealed a significant main effect of presence $\left[F(1,58)=5.80, M S_{\mathrm{e}}=\right.$ $16.172, p=.019$ for accuracy; $F(1,58)=36.46, M S_{\mathrm{e}}=$ $33,247, p<.001$ for speed], with performance being less accurate but faster in the target-present trials. In addition, the male participants were again faster than the females $\left[F(1,58)=10.19, M S_{\mathrm{e}}=143,997, p=.002\right]$. An interaction between gender and target presence revealed that the males were faster mostly in the target-absent trials $\left[F(1,58)=6.89, M S_{\mathrm{e}}=33,247, p=.011\right]$.

More important, the interaction between orientation and gender was significant for accuracy $[F(1,58)=8.01$, $\left.M S_{\mathrm{e}}=15.938, p=.006\right]$ but not for speed $(F<1)$. A graph showing the interaction between gender and orientation (for accuracy) is depicted in Figure 6. The means are presented in Table 1, showing that the females were more accurate in the TR-to-BL (/) diagonal and the males in the other. There was also a significant three-way interaction of orientation, gender, and presence for both accuracy and speed $\left[F(1,58)=6.491, M S_{\mathrm{e}}=16.953, p=\right.$ .014 for accuracy; $F(1,58)=4.165, M S_{\mathrm{e}}=3,713, p=.046$ for speed].

The question arising now was whether or not any of the other individual characteristics was similarly related
Table 1

Performance in the Mixed Block of Experiment 3A: Means (and Standard Deviations) of Median Response Times (in Milliseconds) and Percent Correct for the Two Genders in the Two Orientations

\begin{tabular}{|c|c|c|c|c|c|c|c|c|}
\hline \multirow[b]{3}{*}{ Participants' Gender } & \multicolumn{4}{|c|}{ Response Time } & \multicolumn{4}{|c|}{ Accuracy $(\%)$} \\
\hline & \multicolumn{2}{|c|}{$(\backslash)$} & \multicolumn{2}{|c|}{$(/)$} & \multicolumn{2}{|c|}{ (〉) } & \multicolumn{2}{|c|}{$(/)$} \\
\hline & $M$ & $S D$ & $M$ & $S D$ & $M$ & $S D$ & $M$ & $S D$ \\
\hline Male & 677 & 36.1 & 670 & 34.0 & 97.9 & .7 & 95.6 & .7 \\
\hline Female & 838 & 36.1 & 822 & 34.0 & 97.4 & .7 & 98.0 & .7 \\
\hline
\end{tabular}

to orientation. Analyses similar to that for gender were conducted for eye dominance, head tilt, and handedness. None of the two-way interactions of orientation with these individual characteristics reached significance $(F<1$ in most cases). The only interaction whose $F$ rose above 1 was that of orientation and eye dominance for accuracy $\left[F(1,58)=1.491, M S_{\mathrm{e}}=17.695, p=.227\right]$, with the left-eye-dominant participants being more accurate in the TL-to-BR $(\backslash)$ orientation, but it was obviously weaker than that for gender. The analysis with eye dominance also revealed a significant three-way interaction, but only for accuracy $\left[F(1,58)=5.139, M S_{\mathrm{e}}=\right.$ $17.305, p=.027]$. The correlation between gender and eye dominance was found to be .277 , with left eye dominance being more prevalent in the male than in the female participants. Thus, the interaction between orientation and eye dominance can be explained by that between orientation and gender, rather than vice versa.

Having found the two-way interaction between orientation and gender in the mixed block, we wondered whether a similar interaction would emerge between gender and diagonal of locations, even though it did not

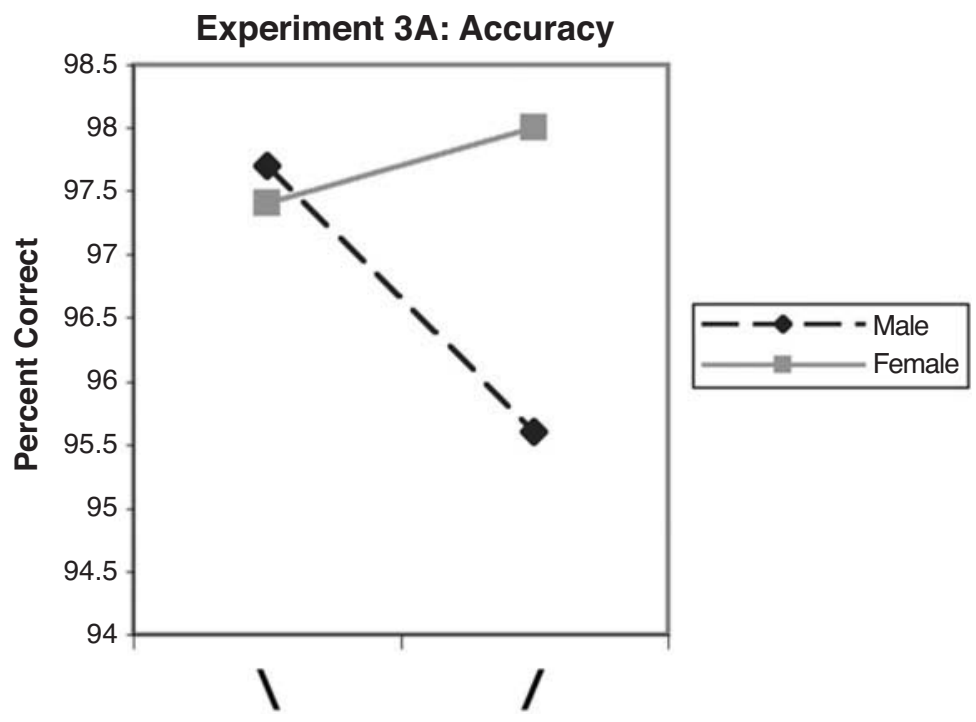

Figure 6. Percent correct responses in the mixed block of Experiment $3 \mathrm{~A}$ in each line orientation, separately for the males and the females. 
appear in the pure blocks, where it was more probable. Therefore, another analysis for the experimental mixed block, focusing on target-present trials only, was conducted, with locations lumped together on the diagonals. Two ANOVAs, one for accuracy and one for speed, with orientation and diagonal of locations as within-participants variables and gender as a between-participants variable, were conducted. No main effect reached significance, but the interaction between orientation and gender was again significant (in the target-present trials only) for accuracy $\left[F(1,58)=11.844, M S_{\mathrm{e}}=10.000, p=.001\right]$ but not for speed $\left[F(1,58)=1.674, M S_{\mathrm{e}}=5,430, p=.201\right]$, with the females being more accurate in the TR-to-BL (/) diagonal. There was no interaction between gender and the diagonals of locations for accuracy or for speed (both $F$ s $<1)$. As to the relation between orientation and diagonals of locations, there was a hint of an interaction for accuracy, with accuracy being higher when the two matched than when they did not $[F(1,58)=3.033$, $\left.M S_{\mathrm{e}}=6.484, p=.087\right]$, but there was none for speed $(F<1)$.

A final analysis of the target-present trials of the experimental mixed block data was focused, separately, on the four pairs of locations at the diagonal ends. Although no differential effect was found for the different diagonals of locations, one could argue that a scan is initiated in one of the end sections and then gets diffused; hence, the lack of interaction between diagonals of locations and gender does not completely rule out an explanation based on location. To further test this, the two locations at each of the four ends of the diagonals were lumped together to form four diagonal ends. Efficiency was different in the different diagonal ends: Performance was fastest and most accurate in the top-left pair of locations and slowest and least accurate in the bottom-right pair. The effect is significant for speed $[F(3,174)=6.439$, $\left.M S_{\mathrm{e}}=12,181, p<.001\right]$ and almost significant for accuracy $\left[F(3,174)=2.518, M S_{\mathrm{e}}=95.625, p=.060\right]$. Importantly, however, performance in the different diagonal ends did not interact with gender $(F<1$ for both speed and accuracy). In other words, the gender-related asymmetry for the diagonals cannot be explained by a differential sensitivity in the different ends.

To summarize, Experiment $3 \mathrm{~A}$ already provided answers to some of our questions: The gender-related perceptual asymmetry was evident also in simple, meaningless stimuli, at least with extended presentation; the asymmetry could be overcome with repeated presentation of similarly oriented stimuli, and, most important, the gender-related asymmetry was found for the orientation of objects but not for their locations. In other words, the orientation hypothesis was corroborated but the location hypothesis was not. Experiment $3 \mathrm{~A}$ also shows that none of the individual characteristics recorded - that is, eye dominance, head tilt, and handedness - can account for the gender asymmetry.

One additional individual characteristic that was not tested in Experiment 3A but could be thought of as me- diating the gender-related asymmetry is hemispheric differences. Specifically, the asymmetry could be mediated by hemispheric differences that are related to visual processing. Such differences ought to be manifested in differential efficiency in visual processing of stimuli in the left and right hemifields. But, to be able to refer to visual fields, eye movements must be precluded. Therefore, in Experiment 3B stimulus presentation was brief- too brief to allow free eye movements.

\section{Experiment 3B}

Experiment 3B was, in fact, a replication of Experiment $3 \mathrm{~A}$ with the exception that stimulus presentation was brief. As such, it could fulfill two objectives: to find out whether the asymmetry between the diagonals is low level or requires free eye movements to develop, and to test the hypothesis that differential sensitivity to the right and left visual hemifields may be related to differential preference for one diagonal or the other.

\section{Method}

The method was almost identical to that of Experiment 3A. The only change was in procedure, with the test display disappearing after $300 \mathrm{msec}$ (pretests indicated that performance is at chance in shorter presentations). A new pool of 67 students, none of whom had participated in Experiment 3A, participated in this experiment. Seven participants whose accuracy in the practice block did not exceed 55\% were excluded from the analysis, leaving 30 males and 30 females. Tests revealed that of the 60 remaining participants 25 were right-eye dominant, 35 were right-hand dominant, 22 had a rightward head tilt, 16 had a leftward tilt, and 22 did not show any tilt.

\section{Results}

Pure blocks. In the pure blocks, the only significant effect was that of presence, with target-present trials being less accurate $\left[F(1,58)=6.571, M S_{\mathrm{e}}=682.539\right.$, $p=.013]$ but faster $\left[F(1,58)=24.297, M S_{\mathrm{e}}=3,646\right.$, $p<.001]$ than trials in which no target appeared. Surprisingly, performance was slightly but consistently faster in the TR-to-BL (/) orientation than in the TL-to$\mathrm{BR}(\backslash)$ orientation $\left[F(1,58)=3.985, M S_{\mathrm{e}}=2,308, p=\right.$ .051 , the mean difference being $12 \mathrm{msec}]$. Gender interacted neither with orientation $[F<1$ for speed and $F(1,58)=1.426, M S_{\mathrm{e}}=220.977, p=.237$ for accuracy $]$ nor with the diagonal of locations $(F<1$ for both speed and accuracy). The only significant interaction was between orientation and diagonal of locations. Here again, the participants were more efficient when the diagonals matched than when they did not. This interaction was significant for accuracy $\left[F(1,58)=4.393, M S_{\mathrm{e}}=81.836\right.$, $p=.040]$ but not for speed $\left[F(1,58)=1.986, M S_{\mathrm{e}}=\right.$ $1,149, p=.164]$.

Mixed block. In the experimental mixed block of Experiment $3 \mathrm{~B}$, responses were again faster $[F(1,58)=$ $15.396, M S_{\mathrm{e}}=5,438, p<.001$ ] and less accurate [though not significantly so; $F(1,58)=3.210, M S_{\mathrm{e}}=137.109, p=$ $.078]$ for target-present than for target-absent trials. More important, the interaction between orientation and gender was again significant for accuracy $[F(1,58)=5.629$, 
$\left.M S_{\mathrm{e}}=51.016, p=.021\right]$. A graph showing this interaction is depicted in Figure 7. The interaction did not reach significance for speed $\left[F(1,58)=2.375, M S_{\mathrm{e}}=1,714, p=\right.$ .129] but was in the same direction as the interaction for accuracy: The female participants were more efficient in the TR-to-BL (/) orientation and the male participants in the opposite orientation (see Table 2).

Similar analyses for accuracy and speed in the mixed block were conducted using the other individual characteristics of eye dominance, head tilt, and handedness, even though this time none of these traits was correlated to gender. None interacted with orientation. In most cases, $F$ was less than 1 and in the few exceptions in which $F$ rose above 1, it did not exceed 1.2.

Further analyses, for diagonal of locations and for the pairs of locations at the four ends of the diagonals (see Experiment $3 \mathrm{~A}$ ), again did not reveal interactions with gender. In the two analyses for diagonal of locations - one for accuracy and one for speed - with orientation, gender, and diagonal of locations as factors, tests of the interaction between diagonal of locations and gender show $F<1$ for accuracy and $F(1,58)=1.045, M S_{\mathrm{e}}=1,773, p=.311$ for speed. The analysis for accuracy did reveal a significant interaction between orientation and diagonal of locations $\left[F(1,58)=11.905, M S_{\mathrm{e}}=30.195, p=.001\right]$, with accuracy being higher when the two were matched.

The analyses with the four pairs of locations at the ends of the two diagonals as factors revealed, as in Experiment $3 \mathrm{~A}$, a main effect of diagonal ends $[F(1,58)=$ $8.971, M S_{\mathrm{e}}=138.320, p<.001$ for accuracy and $F(1,58)=6.347, M S_{\mathrm{e}}=5,792, p<.001$ for speed]. Diagonal ends, however, did not interact with gender (both $\left.F_{\mathrm{S}}<1\right)$.

The other objective of Experiment 3B was to test the hypothesis that hemispheric differences mediated the
Table 2

Performance in the Mixed Block of Experiment 3B: Means (and Standard Deviations) of Median Response Times (in Milliseconds) and Percent Correct for the Two Genders in the Two Orientations

\begin{tabular}{|c|c|c|c|c|c|c|c|c|}
\hline \multirow[b]{3}{*}{ Participants' Gender } & \multicolumn{4}{|c|}{ Response Time } & \multicolumn{4}{|c|}{ Accuracy $(\%)$} \\
\hline & \multicolumn{2}{|c|}{$(\backslash)$} & \multicolumn{2}{|c|}{$(/)$} & \multicolumn{2}{|c|}{ (\) } & \multicolumn{2}{|c|}{$(/)$} \\
\hline & $M$ & $S D$ & $M$ & $S D$ & $M$ & $S D$ & $M$ & $S D$ \\
\hline Male & 615 & 21.4 & 624 & 20.0 & 91.1 & 1.7 & 88.4 & 1.4 \\
\hline Female & 620 & 21.4 & 613 & 20.0 & 87.8 & 1.7 & 89.5 & 1.4 \\
\hline
\end{tabular}

gender-related asymmetry for diagonals. To test this, we went back to the data of the pure blocks and first calculated, for every participant, on which side their accuracy was higher. ${ }^{3}$ This value was used to divide the participants into three groups: a group of left preferrers, who had fewer errors in the left than in the right locations $(N=26)$; a group of right preferrers $(N=14)$; and a group for which the number of errors in the left and right locations did not differ, which was omitted from the analysis. We then conducted a new ANOVA for accuracy in the mixed block, this time only for those who exhibited a side preference (with side preference as a factor instead of gender). Orientation did not interact with side preference $\left[F(1,38)=1.663, M S_{\mathrm{e}}=57.031, p=.205\right]$. A similar analysis, using speed in the pure blocks to split the participants into side preference groups and analyzing the variance of speed with side preference instead of gender, also did not show an interaction between side preference and orientation $(F<1)$.

To summarize, Experiment 3B provides a full replication of Experiment $3 \mathrm{~A}$ and also shows that the genderrelated perceptual asymmetry is early, with the effect present even with brief presentation. However, of the in-

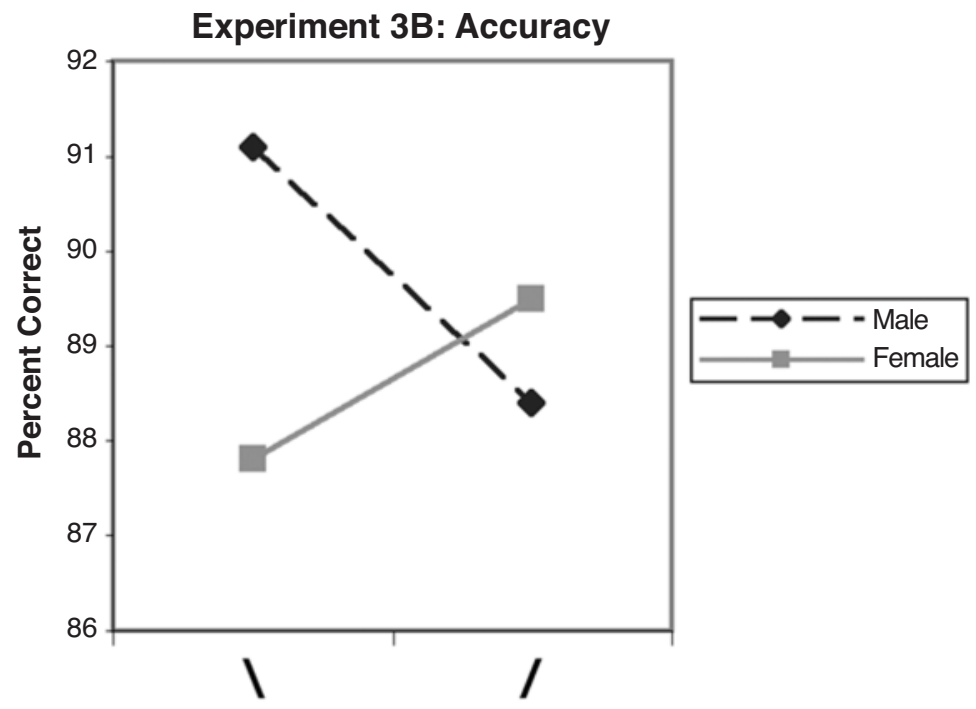

Figure 7. Percent correct responses in the mixed block of Experiment 3B in each line orientation, separately for the males and the females. 
dividual characteristics tested, not even hemispheric differences could explain the interaction between gender and orientation.

\section{Discussion}

The main objective of Experiment 3 was to test the two hypotheses regarding the perceptual asymmetry observed in the first two experiments - namely, whether the asymmetry results from different processing of locations along one diagonal and of those along another, or from different processing of elements in one orientation and of those in another. The results of both parts of Experiment 3 show that the source of the asymmetry is in the orientation of diagonally oriented objects and not in their locations.

Experiment 3 also shows that none of the individual characteristics we considered-namely, eye dominance, head tilt, handedness, and hemispheric differences - can account for the gender-related asymmetry.

In addition, the asymmetry is apparently based on early, low-level processing, since it is manifested for brief as well as extended presentations. Still, when stimuli of constant orientation repeat many times, as was the case in the pure blocks, participants apparently adjust to the orientation - as indicated by several hints to and interaction between elements' orientation and their location on the diagonals - and the asymmetry disappears.

\section{GENERAL DISCUSSION}

The three experiments taken together demonstrate a persisting perceptual asymmetry that is related to gender. The first experiment shows that this asymmetry can intervene in aesthetic preferences, with female observers preferring paintings whose basic structure is along the TR-to-BL (/) diagonal whereas male observers prefer paintings of the other diagonal ( $\backslash$ ). The second experiment shows that the layout of a painting (or, as we should now say, the orientation of the objects in the painting) intervenes in the order in which the painting is processed. As a result, the order of processing of objects in a painting may be different from the order of processing of the same objects in its mirror image. Moreover, the change in the order of processing is opposite for the two genders. Experiment 3 provides a third demonstration of the gender-related perceptual asymmetry along the diagonal orientation. It also narrows the description of the phenomenon, showing that it is related to the diagonal structure of the perceived objects, not to their specific locations in the visual field. In addition, it eliminates a number of potential mediators of the gender-related asymmetry.

In retrospect, since the gender-related asymmetry was found in both parts of Experiment 3 but none of the proposed individual characteristics was found to correlate with gender (with the one exception of eye dominance in Experiment 3A only), there was no reason to expect that they would be strongly related to orientation, but we did not know this in advance. It is true that we did know, from the literature, that neither handedness nor eye dominance was correlated with gender (Arnold-Schulz-Gahmen, Selinski, \& Ehrenstein, 1999), although the two are known to be correlated (McManus, Bryden, \& Bourassa, 1996). We also knew that head tilt was related to eye dominance (Greenberg, 1960), but since the latter was not related to gender there was only a slim chance that the former would be so related. Even the evidence for a relation between gender and visual field dominance - as demonstrated, for example, in asymmetries found in line bisection tasks - is rather inconsequential (Jewell \& McCourt, 2000). Still, we could not rule out the possibility that any of these individual characteristics was independently related to the perceptual asymmetry and that it was accidentally unbalanced within the groups of males and females in the first two experiments.

One should remember that the comparisons in all three experiments involve stimuli and their mirror images. As such, the main manipulation in the experiments never changes the objective level of information in a stimulus. Therefore, differences in efficiency due to the change in layout cannot be attributed to differences in spatial visualization ability, for which gender differences have been found before.

What other candidates could there be? Would it be possible to track differences in life experience with diagonal objects for the two genders, such as, for example, greater familiarity with the slash (/) than the backslash $(\backslash)$ for females in word-processing or heavier use of declining graphs by males? ${ }^{4}$

An important question left open is whether the perceptual asymmetry is related to the direction in which a scene is scanned. It is obvious that the visual field contains more content than that which can be processed at one time. Therefore, different parts must be addressed sequentially. The eyes move from one part of the field to another, bringing the elements of interest into the more acute fovea, and attention sequentially selects some elements in the fields before others. In other words, one scans the field, no doubt, either by moving the eyes or by deploying attention from one part to another, and these two levels are normally correlated. However, although it is clear that a scan does take place, it is extremely difficult to find out if it has a preferred (i.e., a default) direction.

One approach to studying the direction of visual scan is by recording eye movements. Unfortunately, when the eye movements of a person are recorded, that person must be looking at something - at a stimulus, at a displayand, as we have shown here, the characteristics of a display can greatly affect the order of visual processing. Not only effects of the contents of a picture but also those of the goal of the viewer are clearly demonstrated in the recordings of eye movements performed by Yarbus (1967). Similarly, the movement of attention across a display is known to be influenced by the nature of the display, with straight lines, for example, encouraging movement along them (see, e.g., Avrahami, 1999). As a result, 
even if a particular direction of scan is found experimentally, it may be specific to the experimental stimulus and to the type of task used.

Another approach adopted to infer a visual scan is to compare visual efficiency in different parts of the field. This approach capitalizes on the assumption that greater efficiency in one part of the field would indicate that that part is processed before another and, hence, a scan from the former to the latter part can be inferred. But here, too, efficiency is measured for a specific task that involves a specific stimulus. Different parts of the visual field have been shown to excel in different tasks. For example, the left and right visual fields have been shown to differ in their efficiency for the local and global aspects. The right hemifield is better at processing local aspects of the stimulus, and the left is better at processing global aspects (Hellige, 1993). Viewed differently, it can be said that the left visual field is more efficient in holding and comparing several objects at once, whereas the right visual field is more efficient in detecting a single object. With efficiency related to visual task, one can hardly infer a default scan direction from differential efficiency in different parts of the visual field.

Moreover, the scan of a stimulus may be exhaustive. In that case, RT to different parts of the stimulus would not be different even if a scan did take place. Indeed, to infer a scan from the pattern of efficiency, Avrahami (1998) compared efficiency in four locations (top, bottom, left, and right) in either mixed blocks (several possible locations) or pure blocks (target location known in advance). The argument was that if the different efficiency in the different target locations stemmed from differential sensitivity in these locations, then the difference between locations would be most pronounced in the mixed blocks. If, however, the difference stemmed from a scan in a particular direction, and if such a scan is exhaustive unless target location is known in advance, the superiority of locations that lie where the scan is initiated would be most pronounced in the pure blocks. Since greater differences were found in the pure blocks, a scan, from top right to bottom left, was inferred. In retrospect, even this scan might be related to the type of task and the type of displays used in that study.

To summarize, although an explanation of genderrelated perceptual asymmetry based on differential sensitivity to different locations was rejected here, a hypothesis of a differential scan was not. A new line of study is required to find out if males and females differ in their direction of scan and, more precisely, to determine whether the default direction of one's scan - if such a default is found-is related to one's preference for orientation and whether that direction is related to gender. One should remember, though, that even if the default direction of scan is found to correlate both with the preference for orientation and with gender, such correlations would still not provide the causal direction sought: A differential scan, even if found, could be explained by the differential preference for orientation rather than explain it. For the time being, then, what we have is a gender-related aesthetic preference and a gender-related order of visual processing that is related to a gender-different sensitivity to a stimulus's diagonal orientation.

The implications of this phenomenon for art and science are obvious. Designers should be aware of the asymmetry and attempt to balance the two orientations when they address a mixed audience; they may wish to be selective when targeting a single-gender audience. Scientists, in particular students of visual perception, should be careful when choosing tilted stimuli. Such stimuli, if used, should be balanced in the orientation of their diagonals.

An important point about the gender-related perceptual asymmetry is that, although the effects reported here are all statistically significant, they are by no means absolute. Not all females prefer one diagonal, and not all males prefer the other. The history of painting provides abundant examples of male painters who use the "female" diagonal. Giotto, who is regarded as the father of the diagonal structure in Medieval painting, is an important example of such painters. We still believe that there must, therefore, be some other individual dimension responsible for the asymmetry, a dimension that is correlated with gender. At the moment, we do not know what that dimension might be and are left with the mystery of this gender-related perceptual asymmetry for the diagonals.

\section{REFERENCES}

ARnheIm, R. (1982). The power of the center: A theory of composition in the visual arts. Berkeley: University of California Press.

Arnold-Schulz-Gahmen, B. E., Selinski, S., \& Ehrenstein, W. H. (1999). Eye, ear, hand, and foot: Gender differences of individual laterality profiles [Abstract]. In Twenty-seventh Göttingen Neurobiology Conference (Vol. 2, p. 877). Stuttgart: Thieme.

Avrahami, J. (1998). Presence and absence of top and right advantage as an indication of the attentional strategies underlying object selection. Perception, 2, 431-438.

Avrahami, J. (1999). Objects of attention, objects of perception. Perception \& Psychophysics, 61, 1604-1612.

CHRISTMAN, S. D. (1993). Local-global processing in the upper versus lower visual fields. Bulletin of the Psychonomic Society, 31, 275-278.

Enns, J. T., \& Rensink, R. A. (1992). VScope: A general purpose tachistoscope for the Macintosh. Vancouver: Micropsych Software.

Gaffron, M. (1950). Right and left in pictures. Art Quarterly, 13, 312331.

GreenberG, G. (1960). Eye-dominance and head-tilt. American Journal of Psychology, 73, 149-151.

HeLlige, J. B. (1993). Hemispheric asymmetry: What's right and what's left. Cambridge, MA: Harvard University Press.

Jewell, G., \& McCourt, M. E. (2000). Pseudoneglect: A review and meta-analysis of performance factors in line bisection tasks. Neuropsychologia, 38, 93-110.

KossLYN, S. M. (1987). Seeing and imaging in the cerebral hemispheres: A computational approach. Psychological Review, 94, 148-175.

LEVY, J. (1976). Lateral dominance and aesthetic preference. Neuropsychologia, 14, 431-445.

McLaughlin, J. P. (1986). Aesthetic preference and lateral preferences. Neuropsychologia, 24, 587-590.

Mclaughlin, J. P., Dean, P., \& STanley, P. (1982). Aesthetic preference in dextrals and sinistrals. Neuropsychologia, 21, 147-153.

McManus, I. C., Bryden, M. P., \& Bourassa, D. C. (1996). Handedness and eye-dominance: Explaining the association. Brain \& $\mathrm{Cog}$ nition, 30, 357-358.

Rubin, N., Nakayama, K., \& Shapley, R. (1996). Enhanced percep- 
tion of illusory contours in the lower versus upper visual hemifields. Science, 271, 651-653.

SNODGRASs, J. G., \& VANDERWART, M. (1980). A standardized set of 260 pictures: Norms for name agreement, image agreement, familiarity, and visual complexity. Journal of Experimental Psychology: Human Learning \& Memory, 6, 174-215.

YARBus, A. L. (1967). Eye movements and vision. New York: Plenum.

\section{NOTES}

1. The late artist Raaya Redlich, when discussing this issue with the first author, argued that a mirror image reveals a painting's imperfections. 2. The effects on aesthetic preferences of flipping a painting to its mirror image have been studied before (see, e.g., Levy, 1976; McLaughlin, 1986; McLaughlin, Dean, \& Stanley, 1982) but were related to the laterality of the major object of interest in the painting and to handedness, not to diagonality and gender. Still, to rule out an alternative ex- planation for our results based on the location of objects of interest in the painting (although handedness was fully balanced in our gender groups), a new group of 14 participants was asked to indicate where in each (original) painting the "major object of interest" lay: to the right, to the left, or in between; above, below, or in between. We then looked for correlations between the "righthood" of an original painting - as judged by this new group of participants - and its preferences in the male and female participants of Experiment 1. Similarly, we analyzed the "uphood" of an original painting as indicated by the new group and the preferences of the old groups. No correlations were found.

3. The data from the pure blocks were used because they were not confounded with the manipulation of orientation, as were the data of the mixed block.

4. I thank an anonymous reviewer for suggesting these possibilities.

(Manuscript received May 12, 2003;

revision accepted for publication March 11, 2004.) 